\title{
Implied Evidence of the Functional Role of the Rectus Capitis Posterior Muscles
}

Richard C. Hallgren, PhD; Jacob J. Rowan, DO

From the Department of

Physical Medicine and Rehabilitation (Dr Hallgren) and the Department of Osteopathic Manipulative Medicine (Drs Hallgren and Rowan) at Michigan State University College of Osteopathic Medicine in East Lansing.

Financial disclosures: None reported.

Support: This study was supported in part by research grant No. 09-05-586 from the

American Osteopathic Association.

Address correspondence to Richard C. Hallgren, PhD, Department of Physical Medicine and Rehabilitation, Michigan State University College of Osteopathic Medicine, 909 Fee Rd, East Lansing, Ml 48824-6410. Email: hallgren@msu.edu
Context: Osteopathic physicians often target the rectus capitis posterior minor $(\mathrm{RCPm})$ and rectus capitis posterior major (RCPM) muscles when using muscle energy or soft tissue cervical techniques to treat patients with head and neck pain. The RCPm and RCPM muscles are located deep within the posterior occipitoatlantal and atlantoaxial interspaces, respectively.

Objective: To characterize the functional role of RCPm and RCPM muscles by comparing electromyographic (EMG) activation patterns of these muscles with EMG activation patterns of specific flexor and extensor muscles of the head and neck, the sternocleidomastoid (SCM), and the splenius capitis (SC) muscles, respectively.

Methods: Asymptomatic participants were recruited from the Michigan State University College of Osteopathic Medicine student body. Disposable 25-gauge, bipolar fine-wire intramuscular electrodes were used to collect EMG data from the right and left RCPm and RCPM muscles. Surface electrodes were used to collect EMG data from the right and left SCM and SC muscles. Data were collected as participants performed 4 cycles of flexion and extension with an external 4 -lb force applied to the back of the head and the forehead.

Results: In RCPM muscles, EMG activity was significantly greater $(P<.003)$ during flexion and extension of the head and neck when an external force was applied to the back of the head. EMG activity in SCM muscles was significantly greater $(P<.0001)$ during flexion and extension of the head and neck when an external force was applied to the forehead. The authors observed that EMG activity in SC muscles was significantly greater $(P<.015)$ during flexion and extension of the head and neck when an external force was applied to the back of the head. No significant difference was found in EMG activity in RCPm muscles $(P<.834)$ during flexion and extension of the head and neck, regardless of whether the external force was applied to the back of the head or the forehead.

Conclusion: The EMG activation patterns of the RCPm muscles suggest that their functional role may be to stabilize the occipitoatlantal joint by helping maintain congruency of the joint surfaces. In contrast, the EMG activation patterns of the RCPM muscles suggest that their functional role may be to contribute to extension of the head, primarily at the occipitoatlantal and the atlantoaxial joints.

J Am Osteopath Assoc. 2020;120(6):395-403

doi:10.7556/jaoa.2020.061

Keywords: extension, flexion, head and neck, rectus capitis posterior 
$\mathrm{T}$ he rectus capitis posterior (RCP) muscles are among the few muscles in the human body whose functional role has yet to be defined. The RCP muscles comprise the rectus capitis posterior minor $(\mathrm{RCPm})$ and rectus capitis posterior major (RCPM) muscles. Bilateral contraction of RCP muscles has been proposed to contribute to extension of the head at the occipitoatlantal (OA) and the atlantoaxial (AA) joints, while unilateral contraction of the RCPM muscles has been proposed to contribute to ipsilateral rotation and sidebending of the head. ${ }^{1}$ Considering their small size relative to larger superficial muscles acting in parallel with them, RCP muscles would not be expected to significantly contribute to gross movements of the head. The high spindle density in the RCPm and RCPM muscles suggests that they may provide a significant proprioceptive function. RCPm muscles have been shown to have a significantly greater density of slow-twitch fibers, whereas RCPM muscles have been shown to have a significantly greater density of fast-twitch fibers, which suggests that the functional roles of these 2 muscles may be different. ${ }^{2,3}$

The RCP muscles are located deep within the posterior OA and AA interspaces. Osteopathic physicians often target these muscles when using muscle energy ${ }^{4}$ or soft tissue techniques, such as suboccipital release, to treat patients with head and neck pain. The RCPm muscles are interconnected with the pain-sensitive cervical dura mater through a myodural bridge, ${ }^{5}$ and fatty infiltration (FI) of RCPm muscles has been reported in patients with chronic headache. ${ }^{6}$ The cause of FI is unknown, but it alone would not be expected to be the direct cause of headache. Fatty infiltration would be expected to reduce the strength of these muscles and compromise their ability to maintain normal levels of tension within the myodural bridge, which may result in chronic head and neck pain. The osteopathic medical profession has long maintained that structure and function are reciprocally interrelated, and the loss of functionality of any musculoskeletal component will affect other parts of the body.
In 1985, Johnston et $\mathrm{al}^{7}$ quantified range of motion (ROM) in 16 men and used this information to compare participants with normal and symmetric ROM ( $n=5)$ with participants with a reduced and asymmetric ROM $(n=11)$. The authors hypothesized that a significant loss of total primary ROM was an indicator of somatic dysfunction even in the absence of pain. In 1987, Vorro and Johnston ${ }^{8}$ reported that electromyographic (EMG) data collected from 12 electrode sites on each of the same group of participants from the 1985 study $^{7}$ were synchronized with kinematic data. While these results might seem to be intuitive today, this was the first report that objectively supported the concept of somatic dysfunction as a disturbance of reflex control of the human peripheral movement system.

In previous publications using data from a single cohort of asymptomatic participants,, 10 our research team demonstrated that significant activation of RCP muscles occurs during protrusion and retraction of the head from a neutral head posture (NHP) and that levels of activation, expressed as a percentage of maximum voluntary isometric contraction (MVIC), are independent of the direction of head motion. The current study expands on our previous work by quantifying EMG activation levels of the RCP muscles during flexion and extension of the head and neck and comparing these with EMG activation levels of the SCM and SC muscles that are known to be responsible for flexion and extension, respectively. Our rationale was that cervical muscles acting in parallel with each other should have similar EMG activation patterns during flexion and extension movements.

\section{Methods}

\section{Research Design}

We used a $2 \times 2$, within-participants, factorial design to compare activation levels of RCPm, RCPM, SCM, and $\mathrm{SC}$ muscles from a single group of asymptomatic women and men. Four sets of paced flexion and extension of the head were performed with an external force 
of $4 \mathrm{lb}$ applied to the back of each participant's head, followed by the collection of a second data set as participants performed 4 sets of paced flexion and extension with an external force of $4 \mathrm{lb}$ applied to the forehead. ${ }^{11}$ EMG data were recorded from each of 8 muscles (4 left and 4 right) of each participant. For the current study, flexion was defined as any movement of the cervical spine that resulted in the opening of the articular facets, and extension was defined as any movement that resulted in the closing of the facets. It would be expected that without any external loading, activation of the SCM muscles during flexion of the head and neck would be significantly greater than activation of the SC muscles, and that activation of the SC muscles during extension of the head and neck would be significantly greater than activation of SCM muscles. ${ }^{12}$ Applying an external force to the forehead would require increased activation of SCM muscles to maintain an NHP, to flex the head and neck, and to control the rate of extension. Sherrington Law states that this should be accompanied by decreased activation of the SC muscles. ${ }^{13}$ Conversely, applying an external force to the back of the head would require increased activation of SC muscles to maintain an NHP, to extend the head and neck, and to control the rate of flexion.

\section{Study Population}

The study cohort was the same used for a previous study. ${ }^{9}$ Participants were between the ages of 20 and 40 years. ${ }^{14}$ Our inclusion criteria required participants to be free of head and neck pain and to have no significant motion restrictions in the region of the upper cervical spine as determined by an osteopathic physician (J.J.R.). Participants were excluded if they had been involved in a rear-end motor vehicle accident, had upper back or neck surgery, had a pinched nerve in their neck that produced pain radiating down their arm, had a diagnosis of rheumatoid arthritis, lupus, or spondylitis, or had a stroke.

The study was approved by the Michigan State University institutional review board. SigmaStat (SYSTAT Software) 3.5 was used to determine that a minimum of 11 participants were needed to obtain a power of 0.8 with a statistical significance of $\alpha=.05$. We enrolled 7 women and 13 men.

\section{Instrumentation}

A PRseries EWC-40 (Atlantis Engineering) cervical flexion/extension device was used as a test fixture so participants could be repeatedly and reproducibly positioned for the specific tests. Figure 1 shows a simulated participant performed flexion/extension of the head and neck with an external force applied to the back of the

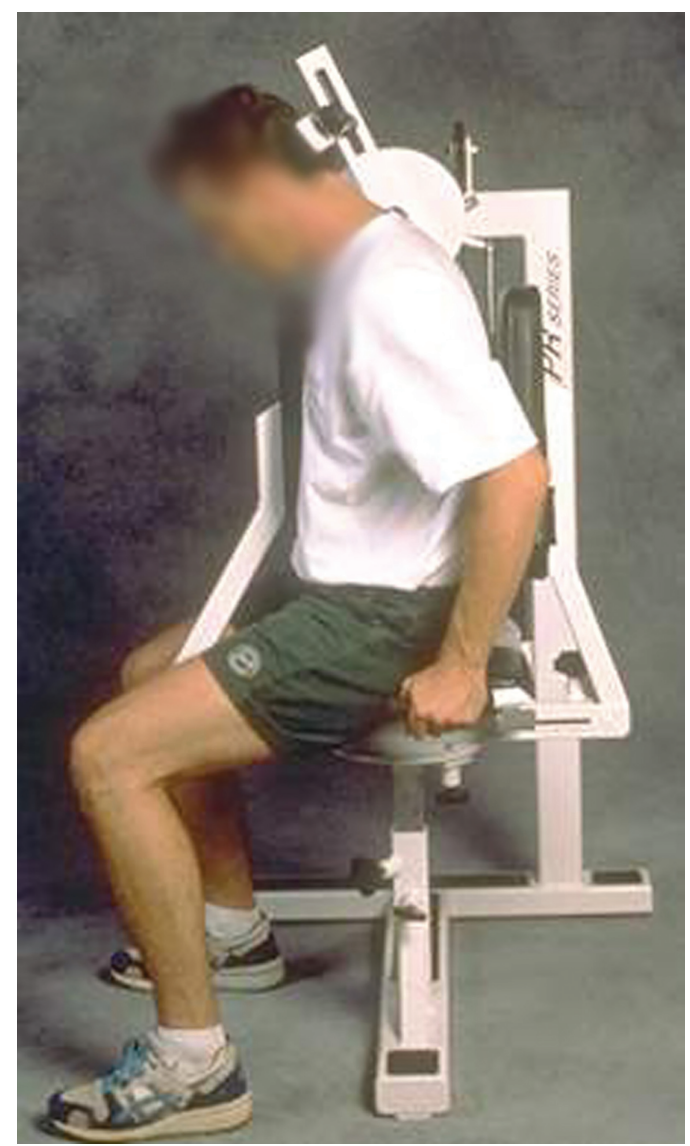

Figure 1.

PRseries EWC-40, cervical flexion/extension device (Atlantis Engineering). Modified with permission from Wolters Kluwer, Inc. Reitman CA, Mauro KM, Nguyen L, Ziegler JM, Hipp JA. Intervertebral motion between flexion and extension in asymptomatic individuals. Spine. 2004;29 (24):2832-2843. doi:10.1097/01.brs.0000147740.69525.58 


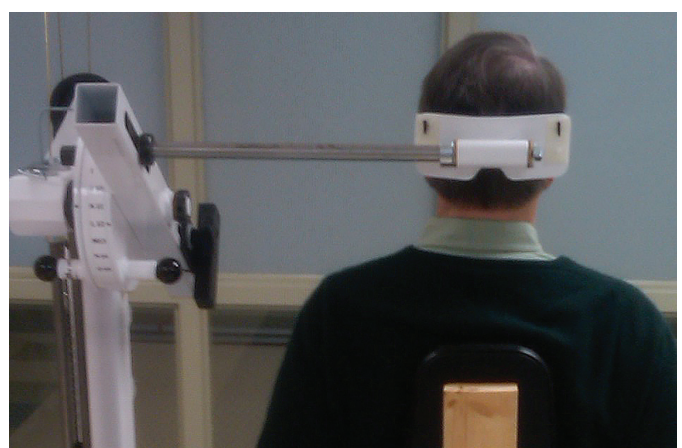

Figure 2.

Posterior view of the cervical flexion/extension device showing the resistance pad used to apply an external force to the back of the head.

head. We adjusted the resistance pad on the cervical device movement arm so that it was at a level just superior to the inion. A head strap held the resistance pad securely to the participant's head. Figure 2 shows the resistance pad from a posterior view of the device. A detailed explanation describing how the device was configured for each participant was described in a previous publication. ${ }^{9}$

Disposable bipolar surface electrodes were placed at the level of C2-3 (Figure 3) to measure EMG voltages from the SC muscles. EMG data were collected from the SCM muscles from surface electrodes that were positioned $20 \mathrm{~mm}$ apart over the distal portion of the muscle belly. ${ }^{15}$ A standardized protocol was used to guide insertion of 25-gauge, disposable, bipolar, stainless steel, fine-wire hooked EMG electrodes into the right and left RCP muscles. ${ }^{16}$ Intramuscular electrodes minimized cross-talk from adjacent muscles and were necessary to quantify EMG voltages from small, deep muscles. After insertion of the electrodes, the external wires were securely taped to the participant's body. The viability of the intramuscular electrodes was assessed. Lack of signal or excessive noise on the signal was noted and corrected when possible.

\section{Processing and Normalization of EMG Data}

Raw EMG voltages were amplified, passed through a bandpass filter (20-1500 Hz), and digitized at 3000

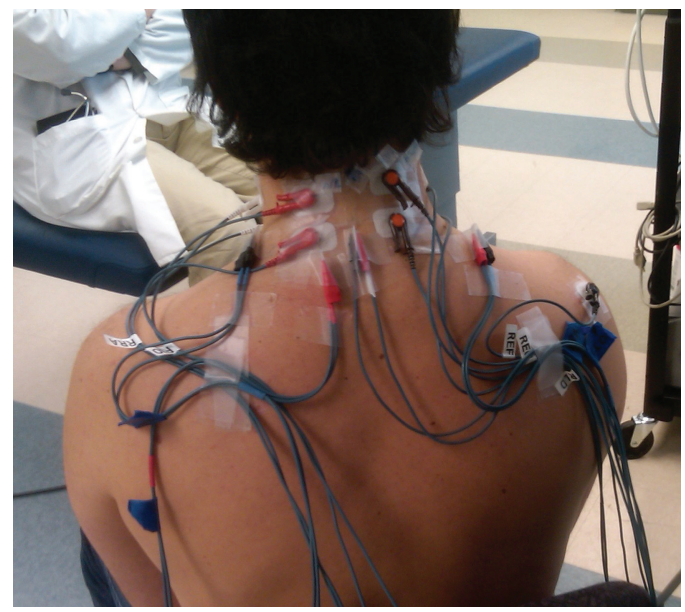

Figure 3.

Placement of intramuscular and surface electrodes.

samples per second. Software written for Matlab (The Mathworks) was used to digitally rectify the data, remove offset voltages and QRS waves, and filter at a low-pass cutoff frequency of $0.5 \mathrm{~Hz}$.

A self-selected NHP was used as a reference posture for the starting position for all tests. ${ }^{17}$ Maximum voluntary isometric contraction (MVIC) voltages were used to normalize muscle activation levels by dividing raw EMG voltage measurements observed during each trial by the maximum EMG voltage that a specific muscle was able to produce and multiplying the result by 100 . A comparison of data among muscles and participants can be accomplished by representing the effort produced by a muscle as a percentage of its maximum capacity. ${ }^{18,19}$ EMG data were collected before the MVIC effort to ensure that each channel of data was free of noise and artifacts. Each of 3 efforts was then held for 5 seconds while data were collected. Participants were allowed to rest for 10 seconds between each effort. For each participant, we treated the maximum value of voltage (MVIC-max) observed across these trials as representative of the maximum voltage that a muscle could generate. Muscle activation values ranged from 0.00195 to 0.98276 . In a previous study, ${ }^{9}$ neither the side of the body nor sex had significant effects on mean muscle activation. The dependent variable was 
then calculated as the mean (SD) value of normalized muscle activation levels of 4 muscles for factors of force direction and head position.

\section{Data Collection}

Before collecting dynamic test data, participants were coached to perform a single cycle of flexion and extension movements of the head during an 8- to 12 -second interval starting from a static NHP (Figure 4A, 4B). Before each effort, EMG data were collected to establish a value for baseline activity/noise and to ensure that each channel of data was free of noise and artifacts. We used a full-cycle protocol to collect 2 sets as participants performed 4 cycles of flexion and extension movements with the resistance pad on the cervical device movement arm applying $4 \mathrm{lb}$ of external force either to the back of the head (Figure 4A) or the forehead (Figure 4B).

The initial direction of motion (flexion or extension) and the direction of the external force were determined randomly. A semicircular array of light-emitting diodes (LEDs) was positioned in the mid-sagittal plane to help pace the participant's rate of motion (Figure 5). ${ }^{20}$ The LEDs were sequenced on and off, and participants were instructed to move their heads as they tracked the LED that was turned on. A full-motion cycle was 10 seconds long. After completing 2 full-motion cycles, the participant was allowed to rest for 15 seconds. The second set of 2 full-motion cycles was repeated with

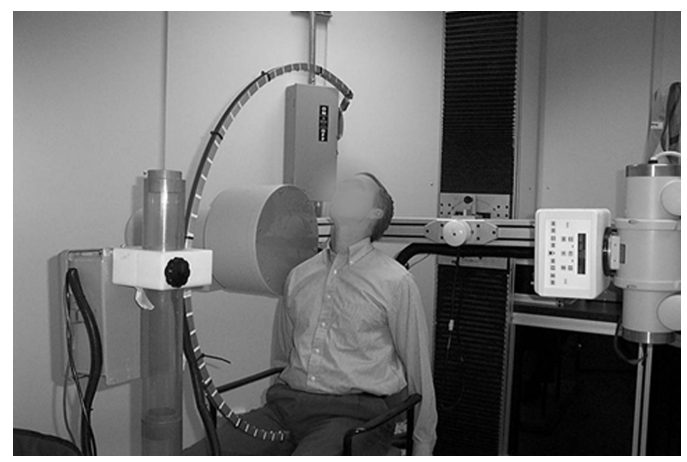

Figure 5.

Light-emitting diode array used to pace the participant's rate of flexion/extension.

the direction of the external force reversed and the participant starting in a direction of motion that was opposite of the first set. After completing all trials, the participant exited the cervical flexion/extension device, was positioned prone, and the electrodes were removed. Each pair of fine-wire electrodes was examined to confirm that all wires had been removed.

\section{Predictors of Muscle Activation}

Application of external force to the forehead or the back of the head was defined as a binary variable, which allowed the comparison of muscle activation levels during flexion and extension movements as a function of force direction. Head motion was also defined as a binary variable, which allowed the comparison of
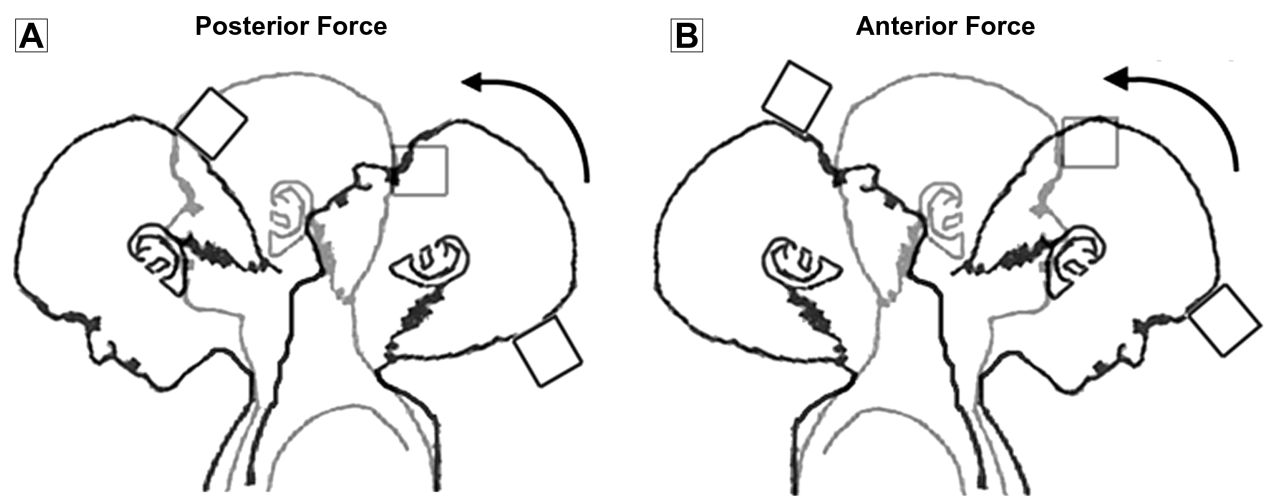

Figure 4.

(A) External force activating splenius capitis muscles. (B) External force activating sternocleidomastoid muscles. 
muscle activation levels with opposing external forces during flexion and extension movements. Neither sex nor body mass index (BMI) was included as a covariate in the statistical analysis. ${ }^{9}$

\section{Data Screening}

Our data screening criteria followed those described in a previous article. ${ }^{22}$ Only 1 of the 20 original participants enrolled in the study was unable to tolerate the insertion of the fine-wire EMG electrodes. All data for 3 participants were excluded from the final analysis because they yielded levels of muscle activation greater than $40 \%$ MVIC-max for some flexion/extension trials, which is an unsustainable long-term, static level of activation. ${ }^{21}$ When an MVIC-max value was very close to 0 , or all voltages recorded from the same electrode were either larger than MVIC-max or all neutral trials resulted in muscle activation greater than $30 \%$ MVIC-max, ${ }^{21}$ it was concluded that the MVIC-max value was inaccurate, and trials associated with that electrode were discarded. One participant yielded levels of muscle activation greater than $100 \%$ MVIC-max. For these participants, we concluded that either the electrodes were not properly placed in the RCP muscles or the equipment malfunctioned. Trials associated with that electrode (eg, all trials on that side of the body for that participant) in any of those situations were discarded. Consequently, the data from the left side for 4 participants and right side for 1 participant were discarded. Four observations were discarded because of implausible values for muscle activation.

\section{Results}

After inaccurate or implausible results were discarded, 257 observations (75\%) from 17 participants (5 women and 12 men) were included in the analysis. The mean (SD) for demographic variables were as follows: weight, 74 (15) kg; height, 177 (12) cm; age, 25 (2) years; and BMI, 24 (4). The observations in this study were not independent because each participant contributed multiple observations to each cell in the within-participants design. Neither side of the body nor head position had significant effects on mean levels of muscle activation. The Table shows mean (SD) levels of muscle EMG activity, as a percentage of the MVIC, for the 4 muscle pairs during flexion and extension movements with external forces applied to either the forehead or the back of the head. Figure 6 shows a box plot of the contents of the Table.

No significant difference was found between EMG activity of RCPm muscles during flexion and extension of the head when either an external force was applied to the forehead or the back of the head $(P=.83)$. EMG activity of RCPM muscles was found to be significantly greater during flexion and extension of the head when an external force was applied to the back of the head than when an external force was applied to the forehead $(P=.003)$.

During both flexion and extension of the head when an external force was applied to the back of the head, EMG activity of SC muscles was significantly greater $(P<.0001)$ than EMG activity of SCM muscles. During both flexion and extension of the head when an external force was applied to the forehead, EMG activity of SCM muscles was significantly greater than EMG activity of SC muscles $(P=.02)$.

\section{Discussion}

A study characterizing the functional role of the RCP muscles is relevant because FI of the RCP muscles has been associated with chronic headache. ${ }^{6}$ Osteopathic physicians often target the RCP muscles when using cervical techniques, such as suboccipital release, to treat patients presenting with head and neck pain.

Activation patterns of RCPM muscles on EMG were found to be similar to SC muscles, implying that RCPM muscles function as extensors. No significant difference was seen between EMG activity of RCPm muscles during flexion and extension of the head when an external force was applied to the forehead or the back of the head. This finding suggests that RCPm muscles cannot be classified as a flexor or as an extensor. Therefore, the RCPm muscles may function as a 


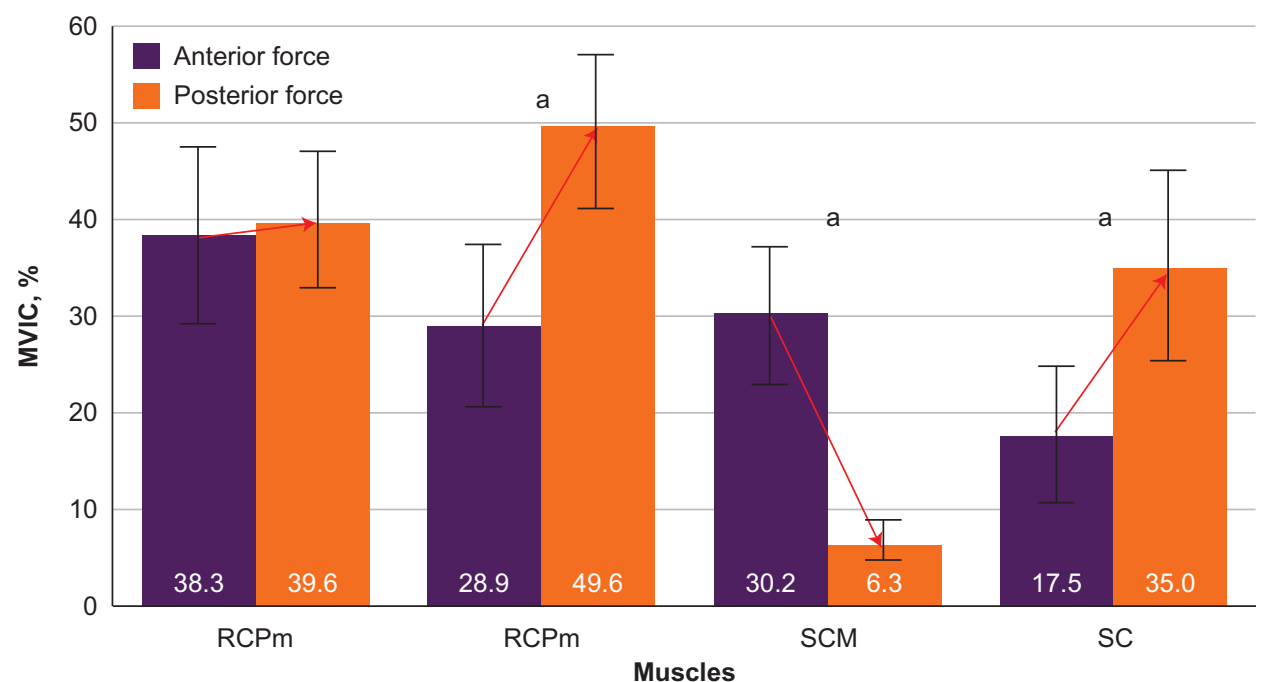

Figure 6.

Electromyographic activity as a percentage of the maximum voluntary isometric contraction (MVIC) and corresponding $95 \% \mathrm{Cls}$ as a function of muscle and the direction of the applied external force. The red arrow associated with each muscle indicates the change in electromyographic values when the force direction is changed from anterior to posterior.

Abbreviations: RCPm, rectus capitis posterior minor; RCPM, rectus capitis posterior major; SC, splenius capitis; SCM, sternocleidomastoid muscles. ${ }^{a}$ Significant statistical difference in muscle activation levels at $P<.05$.

stabilizer of the OA joint. However, these findings do not rule out the possibility that RCPm muscles may also function to maintain normal levels of tension on the dura mater to prevent infolding both during flexion and extension movements of the head and neck. ${ }^{5,23}$

The myodural bridge is a connective link between the RCPm muscles and the dura mater. ${ }^{24-26}$ One study ${ }^{27}$ reported the existence of this connection and added there is also a similar connection between the RCPM muscles and the dura mater. The RCP muscles may function to prevent or limit infolding of the pain-sensitive dura mater during normal movements of the head. ${ }^{5,25}$ Fatty infiltration of RCPm muscles on magnetic resonance imaging has been reported in

Table.

External force applied to the forehead

\begin{tabular}{|c|c|c|c|c|}
\hline Muscle & MVIC, \%, mean (SD) & $95 \% \mathrm{Cl}$ & MVIC, \%, mean (SD) & $95 \% \mathrm{Cl}$ \\
\hline $\mathrm{RCPm}$ & $38.3(17.2)$ & $29.0-47.7$ & $39.6(16.3)$ & $32.5-46.7$ \\
\hline SCM & $30.2(12.7)$ & 23.3-37.1 & $6.3(4.9)$ & $4.2-8.4$ \\
\hline SC & $17.5(13.2)$ & $10.3-24.7$ & $35.0(22.1)$ & $25.3-44.7$ \\
\hline
\end{tabular}

Abbreviations: MVIC, maximum voluntary isometric contraction; RCPm, rectus capitis posterior minor; RCPM, rectus capitis posterior major SC, splenius capitis; SCM, sternocleidomastoid. 
patients who experience chronic headache, with the progression of FI over time directly related to pain and disability. ${ }^{6}$ In general, FI results in a decrease in RCPm muscle mass accompanied by a decrease in patients' ability to generate normal levels of force, and it would compromise their ability to maintain normal levels of tension within the myodural bridge. ${ }^{27}$ The FI could result in clinical manifestations, such as head and neck pain, as a result of dural infolding during daily activities ${ }^{5}$ and could explain the positive effects that some osteopathic manipulative treatment techniques have in managing craniocervical disorders. ${ }^{26}$ The fiber orientation of the myodural bridge is perpendicular to the spinal dura and the RCPm muscles, and it results in a posterior displacement of the dura mater with static loading of the RCPm muscles. ${ }^{5}$ Our study showed that the RCPm muscles are active during both flexion and extension movements of the head and neck, which implies that a functional role of RCPm muscles may be to ensure that appropriate levels of tension are maintained within the myodural bridge to prevent infolding of the pain-sensitive dura mater. $^{5,23,25}$

A loss of RCPm muscle mass due to FI would also decrease the ability of these muscles to generate normal levels of proprioceptive feedback to the central nervous system. In the upper cervical spine, this would compromise the ability to precisely coordinate eye and head movements and respond protectively to sudden external forces. ${ }^{28}$ The incongruence between motor output and sensory feedback could result in an exacerbation of symptoms in patients with whiplash-associated disorders. ${ }^{29}$

Our study had limitations, such as the absence of data from rectus capitis anterior (RCA) muscles. The EMG voltages from RCA muscles might mirror the activation levels of RCPm muscles if their functional role was to increase the stability of the OA joint by helping maintain congruency of joint surfaces. Access to the RCA muscles with fine-wire electrodes was not considered to be feasible because of either an unacceptable level of risk related to hitting a critical vascular structure or because the protocol would not be tolerated by the par- ticipants. Another limitation was the size of the final data set; $25 \%$ of the data were discarded because of dislodged or noisy electrodes and implausible values.

\section{Conclusion}

We found that RCPm muscles were active during flexion and extension movements when an external force was applied to either the forehead or the back of the head, which suggested that the primary functional role of RCPm muscles may be to stabilize the OA joint by helping to maintain congruency of the joint surfaces. The activation patterns of RCPM muscles were similar to activation patterns of the SC muscles, which are responsible for head and neck extension. This finding suggests that RCPM muscles contribute to the extension of the head, primarily at the OA and the AA joints. Future work will continue to investigate the functional significance and clinical relevance of RCP muscles.

\section{Author Contributions}

Both authors provided substantial contributions to conception and design, acquisition of data, or analysis and interpretation of data; both authors drafted the article or revised it critically for important intellectual content; both authors gave final approval of the version of the article to be published; and both authors agree to be accountable for all aspects of the work in ensuring that questions related to the accuracy or integrity of any part of the work are appropriately investigated and resolved.

\section{References}

1. Newell RLM. The back. In: Standing S, ed. Gray's Anatomy. 40th ed. Elsevier Churchill Livingstone; 2008:742-743.

2. Liu JX, Thornell LE, Pedrosa-Domellöf F. Muscle spindles in the deep muscles of the human neck: a morphological and immunocytochemical study. J Histochem Cytochem. 2003;51 (2):175-186. doi:10.1177/002215540305100206

3. Yamauchi M, Yamamoto M, Kitamura K, et al. Morphological classification and comparison of suboccipital muscle fiber characteristics. Anat Cell Biol. 2017;50(4):247-254. doi:10.5115/ acb.2017.50.4.247

4. Pierce-Talsma S, Ji S, Pearce M, Talsma J. Muscle energy for the occipitoatlantal joint. J Am Osteopath Assoc. 2019;119(3):e17-e18. doi:10.7556/jaoa.2019.034

5. Venne G, Rasquinha BJ, Kunz M, Ellis RE. Rectus capitis posterior minor: histological and biomechanical links to the spinal dura mater. Spine. 2017;42(8):e466-e473. doi:10.1097/BRS.0000000000001867 
6. Uthaikhup S, Assapun J, Kothan S, Watcharasaksilp K, Elliott JM. Structural changes of the cervical muscles in elder women with cervicogenic headache. Musculoskelet Sci Pract. 2017;29:1-6. doi:10.1016/j.msksp.2017.02.002

7. Johnston WJ, Vorro J, Hubbard RP. Clinical/biomedical correlates for cervical function: part I. a kinematic study. J Am Osteopath Assoc. 1985;85(7):429-437

8. Vorro J, Johnston WL. Clinical biomechanic correlates for cervical function: part II. a myoelectric study. J Am Osteopath Assoc. 1987;87 (5):353-367.

9. Hallgren RC, Pierce SJ, Prokop LL, Rowan JJ, Lee AS. Electromyographic activity of rectus capitis posterior minor muscles associated with voluntary retraction of the head. Spine J. 2014;14 (1):104-112. doi:10.1016/j.spinee.2013.06.011

10. Hallgren RC, Pierce SJ, Sharma DB, Rowan JJ. Forward head posture and activation of rectus capitis posterior muscles. J Am Osteopath Assoc. 2017;117(1):24-31. doi:10.7556/jaoa.2017.004

11. Pialasse JP, Lafond D, Cantin V, Descarreaux M. Load and speed effects on the cervical flexion relaxation phenomenon. BMC Musculoskel Diord. 2010;11:46. doi:10.1186/1471-2474-11-46.

12. Lindstrøm R, Schomacher J, Farina D, Rechter L, Falla D. Association between neck muscle coactivation, pain, and strength in women with neck pain. Man Ther. 2011;16(1):80-86. doi:10.1016/j.math.2010.07.006

13. Haines DE, ed. Fundamental Neuroscience for Basic and Clinical Applications. Elsevier Inc; 2006.

14. Faulkner JA, Larkin LM, Claflin DR, Brooks SV. Age-related changes in the structure and function of skeletal muscles. Clin Exp Pharmacol Physiol. 2007;34(11):1091-1096. doi:10.1111/j.1440-1681.2007. 04752.x

15. Florencio LL, Ferracni GN, Chaves TC, et al. Analysis of head posture and activation of the cervical neck extensors during a low-load task in women with chronic migraine and healthy participants. $J$ Manipulative Physiol Ther. 2018;41(9):762-770. doi:10.1016/j.jmpt.2018.07.002

16. Hallgren RC, Andary MT, Wyman A, Rowen JJ. A standardized protocol for needle placement in suboccipital muscles. Clin Anat. 2008;21(6):501-508. doi:10.1002/ca.20660

17. Owens EF, Henderson CNR, Gudavalli MR, Pickar JG. Head repositioning errors in normal volunteers: a possible tool to assess the neck's neuromuscular system. Chiropr Osteopat. 2006;14:5. doi:10.1186/1746-1340-14-5

18. Vera-Garcia FJ, Moreside JM, McGill SM. MVC techniques to normalize trunk muscle EMG in healthy women. J Electromyogr Kinesiol. 2010;20(1):10-16. doi:10.1016/j.jelekin.2009.03.010
19. Lehman GJ, McGill SM. The importance of normalization in the interpretation of surface electromyography: a proof of principle. J Manipulative Physiol Ther. 1999;22(7):444-446. doi:10.1016/ s0161-4754(99)70032-1

20. Reitman CA, Mauro KM, Nguyen L, Ziegler JM, Hipp JA. Intervertebra motion between flexion and extension in asymptomatic individuals. Spine. 2004;29(24):2832-2843. doi:10.1097/01. brs.0000147740.69525.58

21. Björkstén M, Jonsson B. Endurance limit of force in long term intermittent static contractions. Scand J Work Environ Health 1977;3 (1):23-27. doi:10.5271/sjweh.2795

22. Hallgren RC, Rowan JJ, Pierce SJ, Bai P, Shafer-Crane GA, Prokop LL. Activation of rectus capitis posterior major muscles during voluntary retraction of the head in asymptomatic subjects. J Manipulative Physiol Ther. 2014;37(6):433-440. doi:10.1016/j. jmpt.2014.07.002

23. Humphreys BK, Kenin SK, Hubbard BB, Cramer GD. Investigation of connective tissue attachments to the cervical spinal dura mater. Clin Anat. 2003;16(2):152-159. doi:10.1002/ca.10109

24. Hack GD, Koritzer RT, Robinson WL, Hallgren RC, Greenman PE. Anatomical relationship between the rectus capitis posterior minor muscle and the dura mater. Spine. 1995;20(23):2484-2486 doi:10.1097/00007632-199512000-00003

25. Nash L, Nicholson H, Lee ASJ, Johnson GM, Zhang M. Configuration of the connective tissue in the posterior atlanto-occipital interspace: a sheet plastination and confocal microscope study. Spine. 2005;30(12):1359-1366. doi:10.1097/01 brs.0000166159.31329.92

26. Palomeque-del-Cerro L, Arráez-Aybar LA, Rodríguez-Blanco $C$ Guzmán-García R, Menendez-Aparicio M, Oliva-Pascual-Vaca Á. A systematic review of the soft-tissue connections between neck muscles and dura mater: the myodural bridge. Spine. 2017;42 (1):49-54. doi:10.1097/BRS.0000000000001655

27. Scali F, Pontell ME, Enix DE, Marshall E. Histological analysis of the rectus capitis posterior major's myodural bridge. Spine J. 2013;13 (5):558-563. doi:10.1016/j.spinee.2013.01.015

28. Lovitt S, Marden FA, Gundogdu B, Ostrowski ML. MRI in myopathy. Neurol Clin. 2004;22(3):509-538. doi:10.1016/j.ncl.2004.03.008

29. Daenen L, Nijs J, Roussel N, Wouters K, Van Loo M, Cras P. Sensorimotor incongruence exacerbates symptoms in patients with chronic whiplash associated disorders: an experimental study. Rheumatology. 2012;51(8):1492-1499. doi:10.1093/rheumatology/ kes050

(c) 2020 American Osteopathic Association

\section{Electronic Table of Contents}

More than 135,000 individuals receive electronic tables of contents (eTOCs) for newly posted content to The Journal of the American Osteopathic Association website. To sign up for eTOCs and other JAOA announcements, visit http://jaoa.org/ss/subscribe.aspx. 